\title{
Experimental evidence of velocity profile inversion in developing laminar flow using MR velocimetry
}

\author{
A. Reci, A. J. Sederman $\dagger$ and L. F. Gladden \\ Department of Chemical Engineering and Biotechnology, University of Cambridge, Philippa \\ Fawcett Drive, Cambridge CB3 0AS, UK
}

(Received xx; revised xx; accepted $\mathrm{xx}$ )

A discrepancy exists between the predictions of analytical solutions of approximate Navier-Stokes equations and numerical finite-difference solutions of the full Navier-Stokes equations regarding the development of laminar flow at the entrance to cylindrical pipes for Newtonian fluids. Starting from a uniform velocity profile at the entrance to the pipe, analytical solutions of approximate Navier-Stokes equations predict the velocity profile to have a maximum at the centre of the pipe at all times. In contrast, numerical finite-difference solutions of full Navier-Stokes equations have suggested that the location of the velocity maximum moves from the wall towards the centre of the pipe in a short distance from the entrance, after which it remains at the centre of the pipe. This study presents the first experimental evidence of the moving velocity maximum from the wall towards the centre of the pipe. The initial uniform velocity profile was achieved by flowing the fluid through a monolith composed of narrow parallel channels and the flow development was investigated using Magnetic Resonance velocimetry. The experimentally observed variation of the position and size of the velocity maximum with the Reynolds number and the distance from the entrance to the pipe is shown to be in good agreement with the predictions of numerical finite-difference solutions of the full Navier-Stokes equations.

\section{Key words:}

\section{Introduction}

The subject of entrance laminar flow in pipes for Newtonian fluids has been extensively studied over the years. The main focus of experimental research in this area to-date has been to establish the entrance length required for fully developed Hagen-Poiseuille flow and the excess pressure drop incurred due to momentum change at the entrance (Rieman 1928; Nikuradse 1950; Goldstein 1938; McComas \& Eckert 1965; Atkinson et al. 1967; Berman \& Santos 1969; Sylvester \& Rosen 1970; Durst et al. 2005). The experimental data have shown that the entrance length required for fully developed Hagen-Poiseuille flow, $z_{\text {ent }}$, is well approximated by $z_{\text {ent }}=0.06 D R e_{\mathrm{p}}$, and that the excess pressure drop incurred at the entrance, $\Delta p_{\text {exc }}$, is well approximated by $\Delta p_{\text {exc }}=0.62 \rho u_{\text {mean }}^{2}$, where $D$ is the diameter of the pipe, $R e_{\mathrm{p}}$ is the Reynolds number based on the pipe diameter, $\rho$ is the density of the fluid and $u_{\text {mean }}$ is the mean velocity. These experimental results are in good agreement with the results of analytical (Langhaar 1942; Collins \& Schowalter

$\dagger$ Email address for correspondence: ajs40@cam.ac.uk 
1962; Campbell \& Slattery 1963; Sparrow et al. 1964; Mohanty \& Asthana 1978) and numerical methods (Hornbeck 1964; Christiansen \& Lemmon 1965; Vrentas et al. 1966; Atkinson et al. 1969; Chen 1973; Gupta 1977; dos Santos \& Figueiredo 2007; Kanda \& Shimomukai 2009).

Given the aforementioned focus, experimental measurements have generally been made at relatively large distances from the entrance to the pipe and a discrepancy between the predictions of analytical and numerical methods about the development of the velocity profile very close to the entrance to the pipe has not been investigated experimentally. Early approaches considering the development of the velocity profile very close to the entrance of the pipe were based on analytical solutions of approximate Navier-Stokes equations obtained by performing linearization of the inertial terms (Boussinesq 1891; Langhaar 1942; Sparrow et al. 1964; Wiginton \& Wendt 1969) or using Prandtl's boundary layer assumptions and solving these approximate equations using integral methods (Schiller 1922; Campbell \& Slattery 1963; Mohanty \& Asthana 1978), series expansions (Collins \& Schowalter 1962; Schlichting 1969; van Dyke 1970; Wilson 1971) or numerical finite-difference methods (Bodoia \& Osterle 1961; Hornbeck 1964; Christiansen \& Lemmon 1965). These approximations were relaxed with the advent of digital computation, which enhanced the capability to solve the full Navier-Stokes equations by numerical methods. Using this approach, various workers reported numerical finite-difference solutions of the development of the velocity profile for laminar flows in a cylindrical pipe (Wagner 1975; Friedmann et al. 1968; Goldberg \& Folk 1988; Dombrowski et al. 1993; Kountouriotis et al. 2016). A salient feature has been captured by the numerical finite-difference solutions of the full Navier-Stokes equations which was not evident in the analytical solutions of approximate Navier-Stokes equations: Starting from a uniform velocity profile, the axial velocity attains a maximum at a position other than the centre of the pipe for a short distance downstream of the flow, with a local minimum at the centre of the pipe. The position of the maximum has been reported to move from the wall towards the centre of the pipe, staying at the centre thereafter. An illustration of the difference between the velocity profile development predicted from analytical solutions of approximate Navier-Stokes equations and numerical finite-difference solutions of full Navier-Stokes equations is given in figure 1.

The phenomenon predicted from the numerical finite-difference solutions of full Navier-Stokes equations is not constrained to the case of a uniform velocity profile at the entrance to the pipe, as was shown in the work of dos Santos \& Figueiredo (2007), who studied numerically the entrance flow in a pipe through a contraction. However, the inlet boundary condition has a large influence on the exact development of the velocity profile (Wagner 1975; Friedmann et al. 1968). The developing axial velocity profile is accompanied by a non-zero radial velocity component, but this is expected to be negligible at $R e_{\mathrm{p}}>10$ for a starting uniform velocity profile (Wagner 1975; Friedmann et al. 1968; Dombrowski et al. 1993). The physical explanation that has been given for the maximum in the axial velocity profile not being at the centre of the pipe (Fargie \& Martin 1971) is that the rate of flow development spreads inwards from the wall towards the centre of the pipe, due to larger shear stresses at the wall. Therefore, the acceleration needed to compensate for the deceleration at the wall is first felt close to the wall, causing a maximum in the axial velocity profile which moves towards the centre of the pipe. Since the phenomenon is constrained to a short distance after the entrance to the pipe, questions have been raised whether this is a truncation artefact of the numerical finite-difference methods (Friedmann et al. 1968; Gillis 1969) and whether it is possible to obtain sufficiently accurate experimental data to validate this behaviour (Atkinson et al. 1969). 

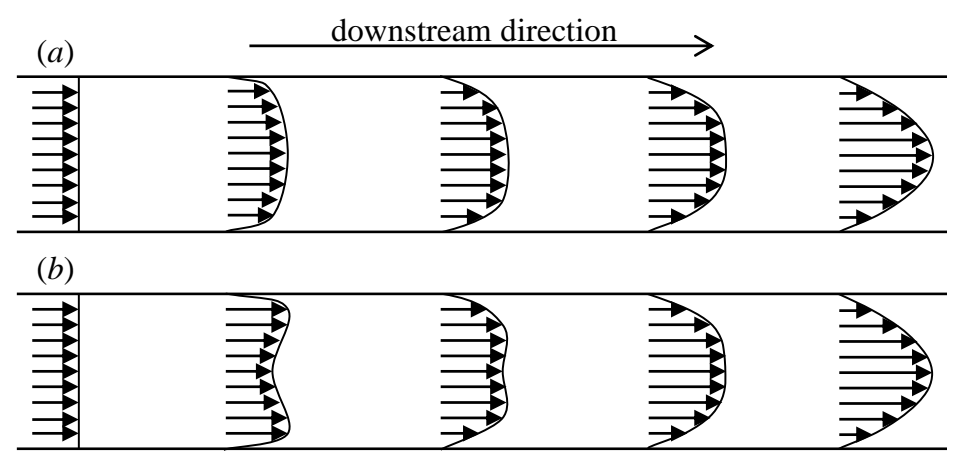

FIgURE 1. Schematic of the development of the axial velocity profile at the entrance to a cylindrical pipe for laminar flow, as predicted by $(a)$ analytical solutions of approximate Navier-Stokes equations and by $(b)$ numerical finite-difference solutions of full Navier-Stokes equations.

This work presents the first experimental study of the axial velocity profile development for laminar flow of a Newtonian fluid very close to the entrance to a cylindrical pipe, with the aim of investigating whether the behaviour of the axial velocity profile is closer to the predictions of analytical solutions of approximate Navier-Stokes equations or to the predictions of numerical finite-difference solutions of full Navier-Stokes equations. The velocity profile at the entrance to a pipe is closely related to the local pressure distribution and heat and mass transfer coefficients. Therefore, the experimentally measured velocity profiles can be used to validate or refute Computational Fluid Dynamics (CFD) results which are commonly used in the design of engineering systems where entrance flow is important, such as capillary rheometers (Mackley \& Rutgers 1998), microfluidic channels (Gervais \& Jensen 2006), filtration equipment (Geraldes et al. 1998), injection moulding (Kuo \& Kamal 1976) and impinging jet systems (Hancock \& Bush 2002). The close relationship between the velocity profile and pressure distribution during developing laminar flow has also been proposed to aid the diagnosis and treatment of blood flow related diseases (Ku 1997; Varghese et al. 2007).

The paper is structured as follows. Section 2 gives a brief overview of Magnetic Resonance (MR) velocimetry, the technique used to measure velocities in this work. The experimental setup and materials used are described in section 3 and the results, discussion and comparison with literature data are presented in section 4 .

\section{MR velocimetry}

The principles of MR velocimetry have been covered in detail by Callaghan (2011). For a review on the applications of MR velocimetry to fluid mechanics problems in particular or to industrially important systems in general, the reader is referred to Fukushima (1999) and Gladden et al. (2006), respectively. Unlike conventional velocity measuring techniques of Laser Doppler Velocimetry (LDV) or Anemometry (LDA), Hot Wire Anemometry (HWA) and Pitot tubes, MR velocimetry is able to measure velocities at different spatial locations simultaneously and is routinely used to measure all three components of the flow field in two dimensional slice images, which can be acquired in any direction, as well as three dimensional volume images. MR velocimetry is non-invasive in the sense that no measuring devices or tracer particles are inserted in the flow, as is the case with the most prominent technique of Particle Image Velocimetry (PIV) or with Doppler Ultrasound Velocimetry (DUV). MR velocimetry can be used to study optically 
opaque systems, such as the velocity inside monolith channels, and gives chemically selective information. The main disadvantages include the inability to study ferromagnetic materials. The historical limitations of MR velocimetry in terms of spatial and temporal resolution have been significantly reduced by advances in hardware, development of ultrafast imaging techniques (Ahn et al. 1986; Hennig 1986) and acqusition methods (Candés et al. 2006; Donoho 2006). It is now possible to acquire sub-millimeter resolution 2D MR velocity images in $<10 \mathrm{~ms}$ (Gladden \& Sederman 2013). Applications of MR velocimetry to fluid mechanics problems include the study of flow past obstructions and in complex geometries (Xia et al. 1992; Elkins et al. 2004; Newling et al. 2004; Mullin et al. 2009), as well as multi-phase flow (Tayler et al. 2012, 2014; Boyce et al. 2016).

In its simplest form, MR velocimetry is a combination of an MR imaging technique with a method of encoding velocity in the pixels of the image. MR imaging consists of applying a sequence of radio frequency (RF) pulses and imaging magnetic field gradients, $\mathbf{G}_{\mathrm{im}}$, which encode the spatial resolution in the MR signal (i.e. the image) such that the acquired signal is given by:

$$
S(t)=\iiint M(\mathbf{r}) \exp \left[\mathrm{i} \gamma \mathbf{G}_{\mathrm{im}} \cdot \mathbf{r} t\right] \mathrm{d} \mathbf{r},
$$

where $\mathbf{r}$ is the position vector, $M(\mathbf{r})$ is the magnitude image, $\gamma$ is the gyromagnetic ratio of the nucleus being imaged and the integration is performed over all space. In the present work, and in the majority of studies, the nucleus observed is ${ }^{1} \mathrm{H}$. With the definition of an inverse space variable, $\mathbf{k}=(2 \pi)^{-1} \gamma \mathbf{G}_{\mathrm{im}} t, S(\mathbf{k})$ and $M(\mathbf{r})$ become a Fourier pair, and the magnitude image can be obtained by Fourier transformation of the signal acquired.

Velocity in a given direction is encoded in the phase of the image by a applying a second set of magnetic field gradients, $\mathbf{G}_{\mathrm{vel}}$, in the direction of the velocity. The phase imparted due to the velocity-encoding gradients is given by:

$$
\phi=\gamma\left(\mathbf{r} \cdot \int \mathbf{G}_{\text {vel }}(t) \mathrm{d} t+\frac{\mathrm{d} \mathbf{r}}{\mathrm{d} t} \cdot \int t \mathbf{G}_{\text {vel }}(t) \mathrm{d} t+\frac{1}{2} \frac{\mathrm{d}^{2} \mathbf{r}}{\mathrm{d} t^{2}} \cdot \int t^{2} \mathbf{G}_{\mathrm{vel}}(t) \mathrm{d} t+\cdots\right) .
$$

The velocity-encoding gradients typically consist of a pair of bipolar gradient pulses (or, equivalently, a pair of gradient pulses of the same polarity separated by a $180^{\circ} \mathrm{RF}$ pulse) with magnitude $g$, duration $\delta$ and time between the centre of bipolar gradient pulses $\Delta$. The bipolar gradient pulses have the property that the zeroth moment of $\mathbf{G}_{\mathrm{vel}}$ (the first term in Eq. 2.2) is zero while the second and higher moments of $\mathbf{G}_{\mathrm{vel}}$ (the third and higher terms in Eq. 2.2) can be neglected compared to the first moment $\mathbf{G}_{\mathrm{vel}}$ (Sederman et al. 2004). Therefore, the phase of the image is related to the velocity of the fluid in the direction of the velocity-encoding gradients, $u$, by:

$$
\phi=\gamma g \delta \Delta u \text {. }
$$

\section{Experimental}

Experiments were performed on a flow loop comprising of a vertical cylindrical pipe of inner diameter $D=16 \mathrm{~mm}$. The flow loop was designed such that a uniform velocity profile at the entrance to the cylindrical pipe was produced by flowing the fluid through a regular square-channel monolith. Water with $0.36 \mathrm{mM} \mathrm{GdCl} 3.6 \mathrm{H}_{2} \mathrm{O}$ added $\left(T_{1} \approx T_{2}=80 \mathrm{~ms}\right.$ ) was used as the flowing fluid. Flow was driven upwards by a Watson-Marlow 505s peristaltic pump, with a dampener used to mitigate flow fluctuations. A $100 \mathrm{~mm}$ long cylindrical cordierite monolith with $0.4 \mathrm{~mm}^{2}$ square channels running throughout its length was inserted in the pipe to obtain a uniform 


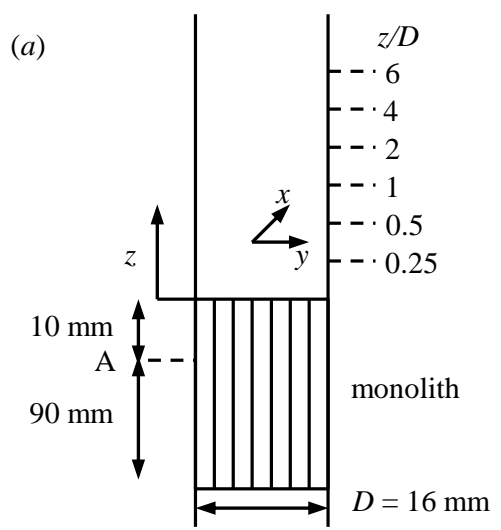

upward flow direction

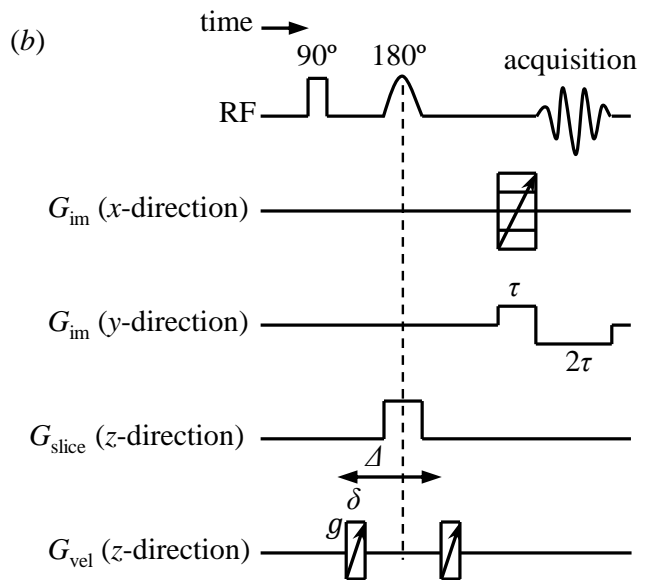

Figure 2. (a) Schematic of the positions inside the monolith (position A) and at the exit of the monolith, or equivalently the entrance to the pipe, $(z / D=0.25,0.5,1,2,4,6)$ where MR velocimetry acquisitions of the axial velocity in a transverse plane were obtained. $(b)$ MR velocimetry pulse sequence used in this work, with the important acquisition parameters identified.

velocity profile. All experiments were conducted on an AV-400 Bruker spectrometer, operating at a resonant frequency of $400.25 \mathrm{MHz}$ for ${ }^{1} \mathrm{H}$ observation, with a radio frequency $(\mathrm{RF})$ coil of $25 \mathrm{~mm}$ diameter. The maximum magnetic field gradient amplitude available in each spatial direction was $146 \mathrm{G} \mathrm{cm}^{-1}$.

MR velocimetry acquisitions of the axial velocity in a transverse slice were performed at a distance of $10 \mathrm{~mm}$ before the exit of the monolith in order to establish whether the velocity profile at the exit of the monolith is uniform and at distances $z$ from the exit of the monolith, $z / D=0.25,0.5,1,2,4,6$, in order to investigate the development of the velocity profile beyond the monolith. The position $z / D=0$ which identifies the exit of the monolith, also identifies the entrance to the pipe. These are illustrated in figure 2(a). Four Reynolds numbers, $R e_{\mathrm{p}}$, were investigated in this study: $120 \pm 10$, $250 \pm 10,500 \pm 20$ and $1100 \pm 50$. The quoted uncertainty, which will be dropped when referring to the Reynolds number in the manuscript, is the calculated standard deviation of the Reynolds numbers measured at the different $z / D$ positions set, and arises from experimental variation in the flow rate at the different positions. The corresponding mean velocities to the Reynolds numbers studied are $0.8,1.6,3.1$ and $6.9 \mathrm{~cm} \mathrm{~s}^{-1}$. The Reynolds number $R e_{\mathrm{p}}$ relates to the flow at the exit of the monolith and uses the diameter of the pipe as the characteristic length. The MR velocimetry technique used was a combination of a conventional spin-echo based imaging technique with velocity-encoding magnetic field gradients, as illustrated in figure $2(b)$. For velocity imaging inside the monolith, the transverse slice thickness was $5 \mathrm{~mm}$, images comprised of $512 \times 512$ pixels and were of resolution $0.039 \mathrm{~mm} \times 0.039 \mathrm{~mm}, \delta=0.25 \mathrm{~ms}, \Delta=0.5 \mathrm{~ms}$, $\tau=0.51 \mathrm{~ms} ; g$ was optimised to achieve a high signal-to-noise ratio, and the time between complex data points acquired in the read $(y)$ direction was $1 \mu \mathrm{s}$. The uncertainty in the pixel-wise velocity measurement inside the monolith was $1.4 \mathrm{~mm} \mathrm{~s}^{-1}$, as calculated from the standard deviation of the velocity image acquired under zero flow conditions. The spatial resolution of the velocity images inside the monolith was high in order to resolve velocities inside individual channels. For all velocity images beyond the exit of the monolith (i.e., within the pipe), the transverse slice thickness was $1 \mathrm{~mm}$, images comprised of $64 \times 64$ pixels and were of resolution $0.313 \mathrm{~mm} \times 0.313 \mathrm{~mm}, \delta=0.5 \mathrm{~ms}$, 


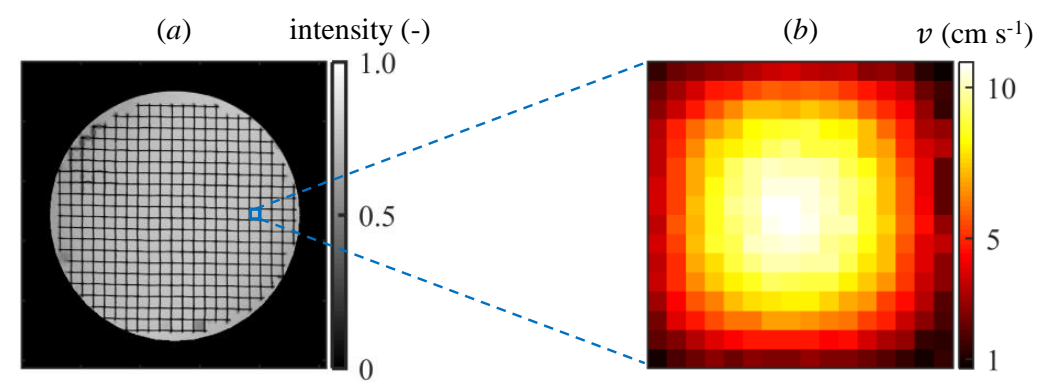

Figure 3. (a) Cross-section magnitude image of the fluid in the monolith, composed of parallel square channels, at a distance of $10 \mathrm{~mm}$ before the exit of the monolith (position A in figure 2(a)). The fluid is MR-active while the monolith is MR-inactive and does not contribute to the MR signal. The image comprises of $512 \times 512$ pixels and is of resolution $0.039 \mathrm{~mm} \times 0.039 \mathrm{~mm}$. The thickness of the wall separating the channels is approximately $0.15 \mathrm{~mm}$. (b) Axial velocity image of a single representative channel highlighted in $(a)$ at $R e_{\mathrm{p}}=500, R e_{\mathrm{c}}=21$. The image comprises of $16 \times 16$ pixels and is of resolution $0.039 \mathrm{~mm} \times 0.039 \mathrm{~mm}$. The maximum uncertainty in the pixel-wise reported velocity is $1.4 \mathrm{~mm} \mathrm{~s}^{-1}$. The axial velocity in the monolith is denoted by $v$ in order to distinguish it from the axial velocity at the exit of the monolith, denoted by $u$.

$\Delta=1 \mathrm{~ms}, \tau=1.28 \mathrm{~ms} ; g$ was optimised to achieve a high signal-to-noise ratio, and the time between complex data points acquired in the read $(y)$ direction was $20 \mu \mathrm{s}$. The uncertainty in the pixel-wise velocity measurement at the exit of the monolith was $0.3 \mathrm{~mm} \mathrm{~s}^{-1}$, as calculated from the standard deviation of the velocity image acquired under zero flow conditions. The phase image used to calculate the velocity in Eq. 2.3 was obtained from the phase difference between two images with positive and negative velocity-encoding gradients $(+g$ and $-g)$ and with correction from a zero velocity image (Sederman et al. 2004).

\section{Results and Discussion}

First, the results of velocity measurements inside the monolith are presented, which show that the velocity profile immediately after the exit of the monolith is well approximated to a uniform velocity profile. Then, the results of the velocity profile development at the entrance to the pipe are given, which show clearly a maximum in the velocity profile whose position moves from the wall to the centre of the pipe with distance along the pipe. These data are then compared to available results from numerical finite-difference solutions of the full Navier-Stokes equations in the literature and the differences are critically discussed.

\subsection{Velocity profile inside the monolith}

In this section, results of the axial velocity measurements on a transverse slice positioned at a distance of $10 \mathrm{~mm}$ before the exit of the monolith are presented. Figure 3( $a$ ) displays the magnitude image of the fluid in the monolith, which shows that the square channels are of similar size and they are distributed evenly throughout the cross-section of the pipe. Each channel comprises of $\sim 16 \times 16$ pixels, which should give sufficient information about the velocity profile inside an individual channel. The velocity profile distribution for an expanded region corresponding to a single representative channel at $R e_{\mathrm{p}}=500$ is given in figure $3(b)$. The Reynolds number in each individual channel, $R e_{\mathrm{c}}$, is estimated to be $\sim 6,10,21$ and 46 when the corresponding Reynolds number at the exit of the monolith, $R e_{\mathrm{p}}$, is $120,250,500$ and 1100 , respectively. $R e_{\mathrm{c}}$ is calculated based on the 


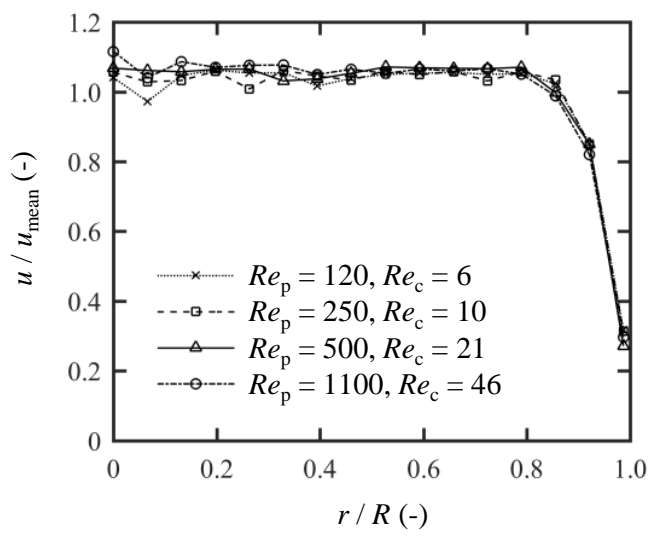

FiguRE 4. Radially averaged axial velocity profile at a position of $10 \mathrm{~mm}$ before the exit of the monolith (position A in figure 2(a)) at the four Reynolds numbers studied. $r$ refers to the radial distance; $R$ refers to the radius of the pipe; and $u_{\text {mean }}$ refers to the mean velocity. The lines are included to guide the eye. The graphs were constructed by radial averaging the mean velocities of the fluid within each channel. The uncertainty related to each data point is dominated by the circular asymmetry of the velocity profile, rather than the uncertainty related to pixel-wise velocity measurements. The maximum uncertainty of the reported $u / u_{\text {mean }}$ values is 0.05 . For reasons of clarity, error bars are not shown.

hydraulic diameter of the channel. At these low Reynolds numbers, the entrance length required for fully developed flow in an individual square channel is $<5 \mathrm{~mm}$ (Sparrow et al. 1967). Therefore, at the imaging section situated $10 \mathrm{~mm}$ before the exit of the monolith (position A in figure 2(a)), fully developed laminar flow is expected in each channel, even at the highest Reynolds number studied. At this distance before the exit of the monolith, the mean velocities in each channel were then radially averaged to give the velocity profile across the whole cross-section of the monolith. The velocity profiles obtained at each of the Reynolds numbers investigated are shown in figure 4 . It is observed that the velocity profile across the cross-section of the monolith is well approximated to a uniform velocity profile at all Reynolds numbers investigated. The slight deviation from the uniform velocity profile close to the wall of the pipe is caused by the non-ideality of the channels close to the wall of the pipe. The non-ideality of the channels extends for approximately one channel side length from the wall of the pipe. This region corresponds to $r / R$ in the range 0.9-1.0, where $r$ is the radial distance from the centre of the pipe and $R$ is the radius of the pipe. It is in this region that the slight deviation from the uniform velocity profile is observed in figure 4 . The effect that the deviation from the uniform velocity profile close to the wall of the pipe has in the development of the velocity profile at the exit of the monolith is discussed in section 4.2. Since the velocity profile at each individual channel is fully developed at $10 \mathrm{~mm}$ before the exit of the monolith, it is expected that the velocity profile across the cross-section of the monolith shown in figure 4 remains the same until the exit of the monolith. Therefore, the velocity profile immediately after the exit of the monolith and entrance to the pipe is well approximated to a uniform velocity profile.

\subsection{Velocity profile development at the entrance to the pipe}

The development of the axial velocity profile at the entrance to the pipe from the initial profile shown in figure 4 is now reported, with a particular focus on the location and magnitude of the maximum velocity in the profile.

The experimental results for the axial velocity profile at distances of $z / D=0.25,0.5,1$, 

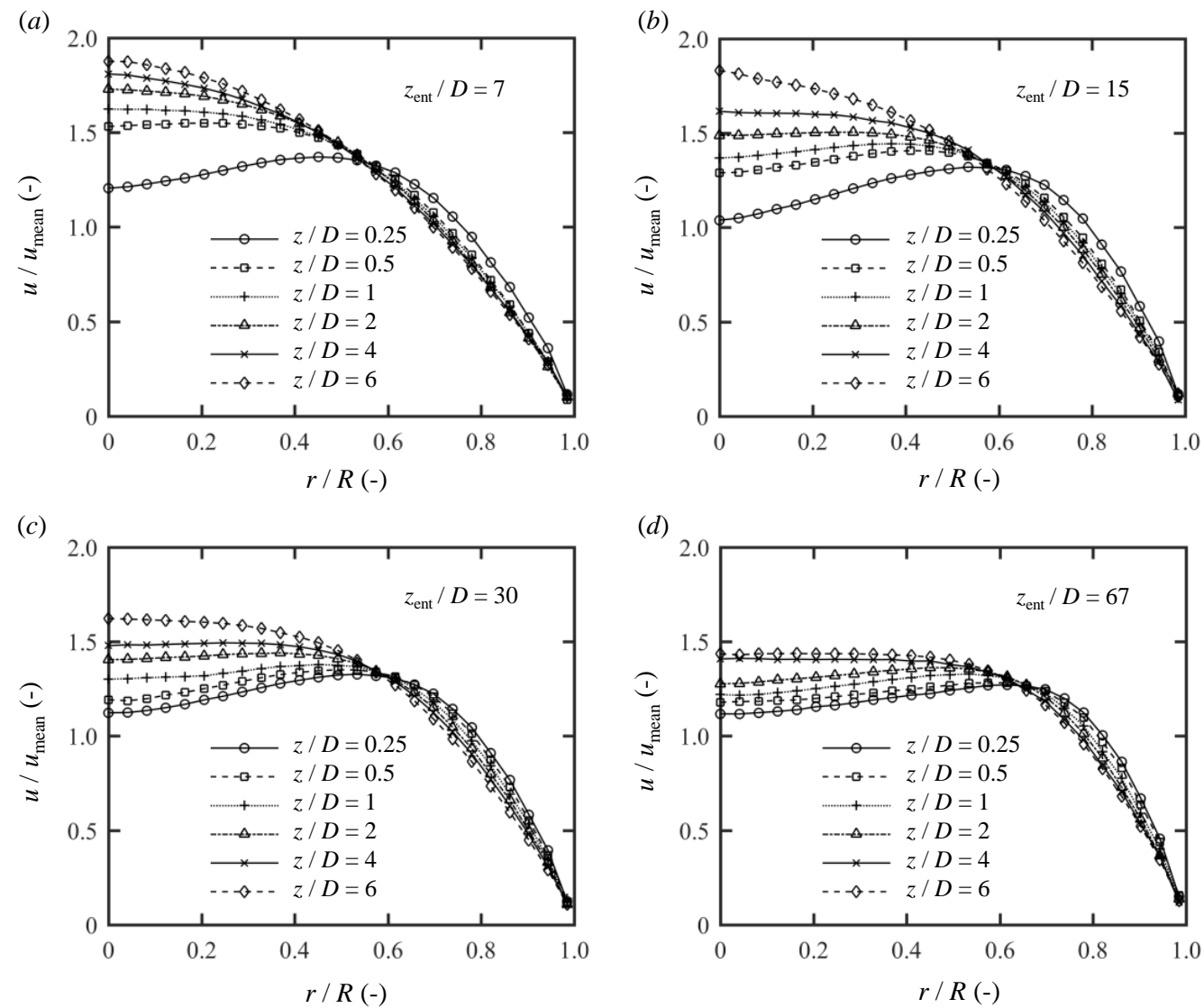

FiguRE 5. Radially averaged axial velocity profiles at positions $z / D=0.25,0.5,1,2,4$ and 6 from the entrance to the pipe and Reynolds numbers, $R e_{\mathrm{p}}$, of $(a) 120,(b) 250,(c) 500$ and $(d)$ 1100. The lines are included to guide the eye. For each Reynolds number, the entrance length required for fully developed laminar flow, $z_{\text {ent }} / D$, is included in the figure. The uncertainty related to each data point is dominated by the circular asymmetry of the velocity profile, rather than the uncertainty related to pixel-wise velocity measurements. The maximum uncertainty of the reported $u / u_{\text {mean }}$ values is 0.03 . For reasons of clarity, error bars are not shown.

2, 4 and 6 from the entrance to the pipe and Reynolds numbers, $R e_{\mathrm{p}}$, of $120,250,500$ and 1100 are presented in figure 5. The key observation from these results is that for a short distance from the entrance to the pipe (the extent of which depends on the Reynolds number), a maximum in the velocity profile which is not positioned at the centre of the pipe is clearly distinguished at all Reynolds numbers investigated. These experimental data therefore strongly support the predictions of the numerical finite-difference solutions of the full Navier-Stokes equations and do not agree with the predictions of analytical solutions of approximated Navier-Stokes equations.

Considering the distance from the entrance of the pipe, $z / D$, over which the phenomenon is visible, it is seen that the phenomenon extends to greater distances down the pipe as $R e_{\mathrm{p}}$ increases; the phenomenon is visible until $z / D \sim 0.5$ for $R e_{\mathrm{p}}=120, z / D \sim 2$ for $R e_{\mathrm{p}}=250, z / D \sim 4$ for $R e_{\mathrm{p}}=500$ and $z / D \sim 6$ for $R e_{\mathrm{p}}=1100$. This observation is in qualitative agreement with the observations of all the works reported which use numerical finite-difference methods to solve the full Navier-Stokes equations and can be explained in terms of the boundary-layer theory (Schlichting 1969); the boundary layer 

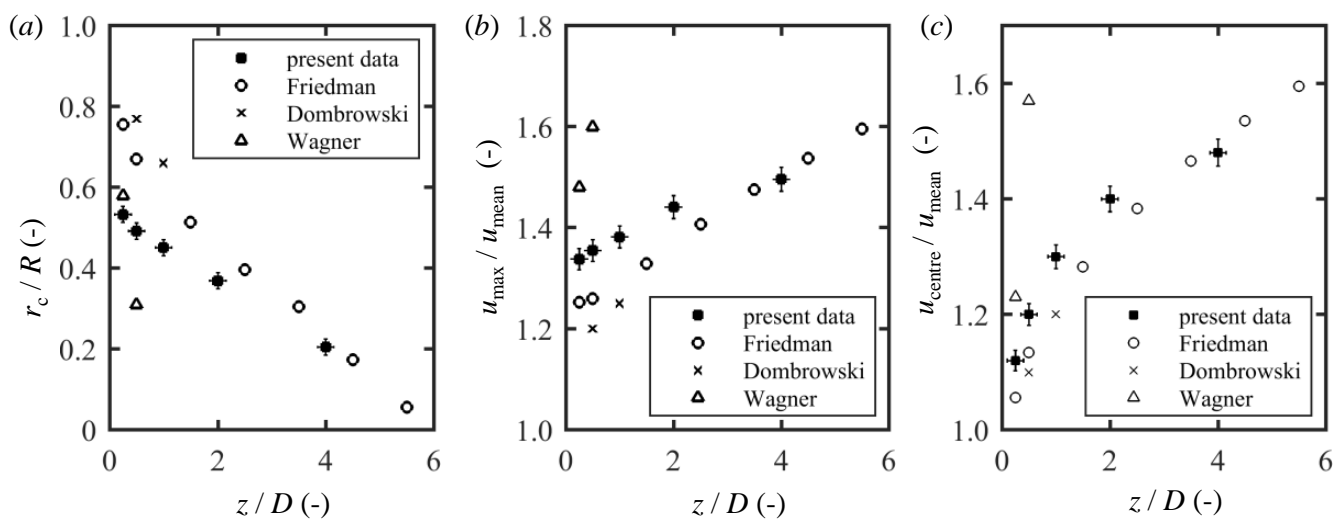

FiguRE 6. Comparison of the present experimental results with the results from finite-difference numerical methods solution of full Navier-Stokes equations from Friedmann et al. (1968) and Dombrowski et al. (1993) at $R e_{\mathrm{p}}=500$ and Wagner (1975) at $R e_{\mathrm{p}}=400$. The graphs show the variation of $(a)$ the critical radius $r_{\mathrm{c}} / R$ at which the maximum of the velocity occurs, $(b)$ the maximum of the velocity $u_{\max } / u_{\text {mean }}$ and $(c)$ the velocity at the centre of the pipe $u_{\text {centre }} / u_{\text {mean }}$ with the distance from the entrance to the pipe $z / D$. The results are only shown for values of $z / D$ at which the maximum in the velocity profile is not at the centre of the pipe. The uncertainty associated with the estimation of $r_{\mathrm{c}} / R$ is dominated by the spatial resolution of the MR image; the uncertainty associated with $u_{\max } / u_{\text {mean }}$ and $u_{\text {centre }} / u_{\text {mean }}$ is dominated by the circular asymmetry of the velocity profile, rather than the uncertainty related to pixel-wise velocity measurements.

develops faster for low Reynolds number, which, as is well known, is why the entrance length for fully developed laminar flow is smaller for low Reynolds numbers.

Sufficient literature data for the quantitative comparison of the present experimental results are only available at $R e_{\mathrm{p}}=500$. Figure 6 shows $(a)$ the variation of the position of the maximum on the velocity profile, $r_{\mathrm{c}} / R,(b)$ the variation of the maximum velocity, $u_{\text {max }} / u_{\text {mean }}$, and $(c)$ the variation of the velocity at the centre of the pipe, $u_{\text {centre }} / u_{\text {mean }}$, with the distance from the entrance to the pipe, $z / D$, at $R e_{\mathrm{p}}=500$ for both the experimental data presented in this study and the results from the numerical finite-difference solutions of the full Navier-Stokes equations of Friedmann et al. (1968), Dombrowski et al. (1993) and Wagner (1975).

Considering the results from the numerical finite-difference solutions of the full Navier-Stokes equations, a major difference is observed between the results of Friedmann et al. (1968) and Dombrowski et al. (1993), as compared to the results of Wagner (1975). This is attributed to the use of different inlet boundary conditions; Friedmann et al. (1968) and Dombrowski et al. (1993) used a uniform velocity profile while Wagner (1975) used inlet boundary conditions corresponding to a piston moving at constant speed and solved the problem in a frame of reference in which the piston is stationary. The observation that the use of different inlet boundary conditions leads to significant quantitative differences in the measured $r_{\mathrm{c}} / R, u_{\text {max }} / u_{\text {mean }}$ and $u_{\text {centre }} / u_{\text {mean }}$ is the main reason for not including in figure 6 the data from the numerical work of dos Santos \& Figueiredo (2007). A minor difference is observed even between the results of Friedmann et al. (1968) and Dombrowski et al. (1993), showing that even with the use of the same inlet boundary conditions, different implementations of numerical methods can lead to different results, in terms of the predicted values of $r_{\mathrm{c}} / R, u_{\max } / u_{\text {mean }}$ and $u_{\text {centre }} / u_{\text {mean }}$.

The inlet boundary condition in the present experimental work is closer to the inlet boundary conditions used by Friedmann et al. (1968) and Dombrowski et al. (1993), 
and it is observed from figure 6 that there is good agreement between the present experimental data and the results of those workers. However, the experimentally observed $r_{\mathrm{c}} / R$ is consistently lower, while $u_{\max } / u_{\text {mean }}$ and $u_{\text {centre }} / u_{\text {mean }}$ are consistently higher than the values predicted by Friedmann et al. (1968) and Dombrowski et al. (1993). These discrepancies can be explained by the fact that the inlet boundary condition in the experiment was not a perfect uniform velocity profile (as it is assumed in the work of Friedmann et al. (1968) and Dombrowski et al. (1993)) but was as shown in figure 4. The consistently lower $r_{\mathrm{c}} / R$ observed experimentally is consistent with the flow already spreading inwards from the wall towards the centre of the pipe before the flow exits the monolith and enters the pipe; this is supported by the data shown in figure 4 . The consistently higher $u_{\max } / u_{\text {mean }}$ and $u_{\text {centre }} / u_{\text {mean }}$ follow from continuity.

\section{Conclusions}

The first experimental evidence of a maximum in the velocity profile positioned not at the centre of the pipe during laminar flow development of a Newtonian fluid at the entrance to a cylindrical pipe has been presented. This phenomenon has been previously reported from numerical finite-difference solutions of the full Navier-Stokes equations, but has not been captured by previous analytical solutions of approximated Navier-Stokes equations or any other experimental study. The experimentally observed behaviour of the phenomenon as a function of the Reynolds number and distance downstream from the entrance to the pipe is in good agreement with numerical finite-difference solutions of the full Navier-Stokes equations. The minor differences can be explained in terms of the use of slightly different inlet boundary conditions.

A. Reci acknowledges Gates Trust Cambridge for financial support. The authors thank Johnson Matthey plc for the provision of the monolith.

\section{REFERENCES}

Aнn, C. В., Kiм, J. H. \& Chо, Z. H. 1986 High-speed spiral-scan echo planar NMR imaging - I. IEEE Trans. Med. Imaging MI-5, 2-7.

Atkinson, B., Brocklebank, M. P., Card, C. C. H. \& Smith, J. M. 1969 Low Reynolds number developing flows. AIChE J. 15, 548-553.

Atkinson, B., Kemblowski, Z. \& Smith, J. M. 1967 Measurements of velocity profile in developing liquid flows. AIChE J. 13, 17-20.

Berman, N. S. \& Santos, V. A. 1969 Laminar velocity profiles in developing flows using a Laser Doppler technique. AIChE J. 15, 323-327.

Bodoia, J. R. \& Osterle, J. F. 1961 Finite difference analysis of plane Poiseuille and Couette flow developments. Appl. Sci. Res. 10, 265-276.

BoussinesQ, J. 1891 Calcul de la moindre longeur que doit avoir un tube circulaire, èvasè á son entrèe, pour qu'un règime sensiblement uniforme s'y ètablisse, et de la dèpense de charge qu'y entraine l'ètablissement de ce règime. Comptes Rendus 113, 49-51.

Boyce, C. M., Rice, N. P., Ozel, A., Davidson, J. F., Sederman, A. J., Gladden, L. F., Sundaresan, S., Dennis, J. S. \& Holland, D. J. 2016 Magnetic resonance characterization of coupled gas and particle dynamics in a bubbling fluidized bed. Phys. Rev. Fluids 1, 074201.

Callaghan, P. T. 2011 Translational Dynamics and Magnetic Resonance. Oxford University Press.

Campbell, W. D. \& Slattery, J. C. 1963 Flow in the entrance region of a tube. J. Basic Eng. 85, 41-46.

Candés, E. J., Romberg, J. \& TaO, T. 2006 Robust uncertainty principles: Exact signal 
reconstruction from highly incomplete frequency information. IEEE Trans. Inf. Theory 52, 489-509.

Chen, R. Y. 1973 Flow in the entrance region at low Reynolds numbers. J. Fluids Eng. 95, $153-185$.

Christiansen, E. B. \& Lemmon, H. E. 1965 Entrance region flow. AiChE J. 11, 995-999.

Collins, M. \& Schowalter, W. R. 1962 Laminar flow in the inlet section of a straight channel. Phys. Fluids 5, 1122-1124.

Dombrowski, N., Foumeny, E. A., Ookawara, S. \& Riza, A. 1993 The influence of Reynolds number on the entry length and pressure drop for laminar pipe flow. Can. J. Chem. Eng. $\mathbf{7 1}, 472-476$.

Donoho, D. L. 2006 Compressed Sensing. IEEE Trans. Inf. Theory 52, 1289-1306.

Durst, F., Ray, S., Ünsal, B. \& Bayoumi, O. A. 2005 The development lengths of laminar pipe and channel flows. J. Fluids Eng. 127, 1154-1160.

VAN Dyke, M. 1970 Entry flow in a channel. J. Fluid Mech. 44, 813-823.

Elkins, C. J., Markl, M., Iyengar, A., Wicker, R. \& Eaton, J. K. 2004 Full-field velocity and temperature measurements using magnetic resonance imaging in turbulent complex internal flows. Int. J. Heat Fluid Flow 25, 702-710.

Fargie, D. \& Martin, B. W. 1971 Developing laminar flow in a pipe of circular cross-section. Proc. Roy. Soc. Lon. A 321, 461-476.

Friedmann, M., Gillis, J. \& Liron, N. 1968 Laminar flow in a pipe at low and moderate Reynolds numbers. Appl. Sci. Res. 19, 426-438.

Fukushima, E. 1999 Nuclear magnetic resonance as a tool to study flow. Annu. Rev. Fluid Mech. 31, 95-123.

Geraldes, V. M., Semiño, V. A. \& Pinho, M. N. 1998 Nanofiltration mass transfer at the entrance region of a slit laminar flow. Ind. Eng. Chem. Res. 37, 4792-4800.

Gervais, T. \& Jensen, K. F. 2006 Mass transport and surface reactions in microfluidic systems. Chem. Eng. Sci. 61, 1102-1121.

Gillis, J. 1969 Viscous flow in the entry region of a straight channel. Phys. Fluids 12, II-280.

Gladden, L. F., Akpa, B. S., Anadon, L. D., Heras, J. J., Holland, D. J., Mantle, M. D., Matthews, S., Mueller, C., Sains, M. C. \& Sederman, A. J. 2006 Dynamic MR imaging of single- and two-phase flow. Trans. IChemE Part A 84, 272-281.

Gladden, L. F. \& Sederman, A. J. 2013 Recent advances in flow MRI. J. Magn. Reson. 229, $2-11$.

GoldBerg, I. S. \& Folk, R. T. 1988 Solutions for steady and nonsteady entrance flow in a semi-infinite circular tube at very low Reynolds numbers. SIAM J. Appl. Math. 48, 770-791.

Goldstein, S. 1938 Modern Developments in Fluid Dynamics. Dover Publications.

Gupta, R.C . 1977 Laminar flow in the entrance of a tube. Appl. Sci. Res. 33, 1-10.

Hancock, M. J. \& Bush, J. W. M. 2002 Fluid pipes. J. Fluid Mech. 466, 285-304.

Hennig, J. 1986 RARE imaging: A fast imaging method for clinical MR. Magn. Reson. Med. 3, 823-833.

Hornbeck, R. W. 1964 Laminar flow in the entrance region of a pipe. Appl. Sci. Res. 13, $224-232$.

Kanda, H. \& Shimomukai, K. 2009 Numerical study of pressure distribution in entrance pipe flow. J. Complexity 25, 253-267.

Kountouriotis, Z., Philippou, M. \& Georgiou, G. C. 2016 Development lengths in Newtonian Poiseuille flows with wall slip. Appl. Math. Comp. 291, 98-114.

Ku, D. N. 1997 Blood flow in arteries. Annu. Rev. Fluid Mech. 29, 399-434.

Kuo, Y. \& Kamal, M. R. 1976 The fluid mechanics and heat transfer of injection mold filling of thermoplastic materials. AIChE J. 22, 661-669.

LANGHAaR, H. L. 1942 Steady flow in the transition length of a straight tube. Trans. ASME: J. Appl. Mech. 9, 55-58.

Mackley, M. R. \& Rutgers, R. P. G. 1998 Capillary Rheometry. In Rheological Measurements (ed. A. A. Coyller \& D. W. Clegg), pp. 167-189. Springer.

McComas, S. T. \& Eckert, E. R. G. 1965 Laminar pressure drop associated with the continuum entrance region and for slip flow in a circular tube. J. Appl. Mech. 32, 765-770. 
Mohanty, A. K. \& Asthana, S. B. L. 1978 Laminar flow in the entrance region of a smooth pipe. J. Fluid Mech. 90 (3), 433-447.

Mullin, T., Seddon, J. R. T., Mantle, M. D. \& Sederman, A. J. 2009 Bifurcation phenomena in the flow through a sudden expansion in a circular pipe. Phys. Fluids 21, 014110 .

Newling, B., Poirier, C. C., Zhi, Y., Rioux, J. A., Coristine, A. J., Roach, D. \& BALCOM, B. J. 2004 Velocity imaging of highly turbulent flow. Phys. Rev. Lett. 93, 154503.

NikuRAdSE, J. 1950 Laws of flow in rough pipes. Tech. Rep. 1292. NASA Tech. Mem.

Rieman, W. 1928 The value of the Hagenbach factor in the determination of viscosity by the efflux method. J. Am. Chem. Soc. 50, 46-55.

dos Santos, R. G. \& Figueiredo, J. R. 2007 Laminar elliptic flow in the entrance region of tubes. J. Braz. Soc. of Mech. Sci. Eng. 29, 233-239.

Schiller, L. 1922 Die entwicklung der laminaren geschwindigkeitsverteilung. Z. Angew. Math. Mech. 2, 96-106.

Schlichting, H. 1969 Boundary-Layer Theory, 7th edn. McGraw-Hill.

Sederman, A. J., Mantle, M. D., Buckley, C. \& Gladden, L. F. 2004 MRI technique for measurement of velocity vectors, acceleration, and autocorrelation functions in turbulent flow. J. Magn. Reson. 166, 182-189.

Sparrow, E. M., Hixon, C. W. \& Shavit, G. 1967 Experiments on laminar flow development in rectangular ducts. J. Basic Eng. 89, 116-123.

Sparrow, E. M., Lin, S. H. \& Lundgren, T. S. 1964 Flow development in the hydrodynamic entrance region of tubes and ducts. Phys. Fluids 7, 338-347.

Sylvester, N. D. \& Rosen, S. L. 1970 Laminar flow in the entrance region of a cylindrical tube: Part I. Newtonian fluids. AIChE J. 16, 964-966.

Tayler, A. B., Benning, M., Sederman, A. J., Holland, D. J. \& Gladden, L. F. 2014 Ultrafast magnetic-resonance-imaging velocimetry of liquid-liquid sustems: Overcoming chemical-shift artifacts using compressed sensing. Phys. Rev. E 89, 063009.

Tayler, A. B., Holland, D. J., Sederman, A. J. \& Gladden, L. F. 2012 Exploring the origins of turbulence in multiphase flow using Compressed Sensing MRI. Phys. Rev. Lett. 108, 264505.

Varghese, S. S., Frankel, S. H. \& Fischer, P.F. 2007 Direct numerical simulation of stentotic flows. Part 1. Steady flow. J. Fluid Mech. 582, 253-280.

Vrentas, J. S., Duda, J. L. \& Bargeron, K. G. 1966 Effect of axial diffusion of vorticity on flow development in circular conduits: Part I. Numerical solutions. AIChE J. 12, 837-844.

WaGner, M. H. 1975 Developing flow in circular conduits: transition from plug flow to tube flow. J. Fluid Mech. 72, 257-268.

Wiginton, C. L. \& Wendt, R. L. 1969 Flow in the entrance region of ducts. Phys. Fluids 12, $465-466$.

Wilson, S. D. R. 1971 Entry flow in a channel. Part 2. J. Fluid Mech. 46, 787-799.

Xia, Y., Callaghan, P. T. \& Jeffrey, K. R. 1992 Imaging velocity profiles: Flow through an abrupt contraction and expansion. AIChE J. 38, 1408-1420. 